\title{
Towards Collaborative Machine Learning Driven Healthcare Internet of Things
}

\author{
Bahar Farahani \\ b_farahani@sbu.ac.ir \\ Faculty of Computer Science and \\ Engineering Shahid Beheshti \\ University
}

\author{
Mojtaba Barzegari \\ mojtaba.barzegari@student.kuleuven.be \\ Biomechanics Section, Department of \\ Mechanical Engineering, KU Leuven
}

\author{
Fereidoon Shams Aliee \\ f_shams@sbu.ac.ir \\ Faculty of Computer Science and \\ Engineering Shahid Beheshti \\ University
}

\begin{abstract}
The relationship between technology and healthcare due to the rise of Intelligent Internet of Things (IoT) and the rapid public embracement of medical-grade wearables has been dramatically transformed in the past few years. Powered by IoT, technology brought disruptive changes and unique opportunities to the healthcare industry including personalized services, tailored content, improved availability and accessibility, and cost-effective delivery. Despite these exciting advancements in transition from clinic-centric to patient-centric healthcare, many challenges still need to be tackled. The key to successfully unlock and enable this digital shift is adopting a holistic architecture to provide high-level of quality in attributes such as latency, availability, and real-time analytics processing. In this paper, we discuss applicability of Intelligent IoT based on Collaborative Machine Learning in healthcare and medicine by presenting a holistic multi-layer architecture. This solution enables real-time actionable insights which ultimately improves decision-making powers of patients and healthcare providers. The feasibility of such architecture is investigated by a case study, ECG-based arrhythmia detection, based on deep learning and Convolutional Neural Network (CNN) methods distributed across endpoint IoT Devices, Edge (Fog) nodes, and Cloud servers.
\end{abstract}

\section{CCS CONCEPTS}

- Computer systems organization $\rightarrow$ Real-time systems; Embedded systems.

\section{KEYWORDS}

Internet of Things, Machine Learning, Health

\section{ACM Reference Format:}

Bahar Farahani, Mojtaba Barzegari, and Fereidoon Shams Aliee. 2019. Towards Collaborative Machine Learning Driven Healthcare Internet of Things. In INTERNATIONAL CONFERENCE ON OMNI-LAYER INTELLIGENT SYSTEMS (COINS), May 5-7, 2019, Crete, Greece. ACM, New York, NY, USA, 7 pages. https://doi.org/10.1145/3312614.3312644

Permission to make digital or hard copies of all or part of this work for personal or classroom use is granted without fee provided that copies are not made or distributed for profit or commercial advantage and that copies bear this notice and the full citation on the first page. Copyrights for components of this work owned by others than the author(s) must be honored. Abstracting with credit is permitted. To copy otherwise, or republish, to post on servers or to redistribute to lists, requires prior specific permission and/or a fee. Request permissions from permissions@acm.org. COINS, May 5-7, 2019, Crete, Greece

(c) 2019 Copyright held by the owner/author(s). Publication rights licensed to ACM. ACM ISBN 978-1-4503-6640-3/19/05 ..\$15.00

https://doi.org/10.1145/3312614.3312644

\section{INTRODUCTION}

The healthcare and technology industries have been highly interlinked for a long time. However, Intelligent Internet of Things (IoT) tsunami is taking increasingly a big leap in almost all healthcare processes. The popularity of miniature wearable devices, public embracement of Artificial Intelligence (AI) and Machine Learning (ML) as well as the rapid growth of Big Data Analytics are creating promising opportunities and unique prospects in customized healthcare services.

The integration of IoT and Cloud Computing has created a paradigm, the Cloud-based Internet of Things, to partially resolve some of the major challenges of IoT such as limited processing capabilities and storage. Over past few years, many players have also started to replace traditional business intelligence tools for processing health data. In this context, companies have started leveraging artificial intelligence-in particular, machine learning-into their eHealth application.

In Cloud-based IoT eHealth model, the massive amount of data needs to be transferred from the devices to the Cloud, demands a considerable amount of available communication bandwidth. Moreover, Cloud Computing incurs large latency due to data exchange between Cloud servers and devices. Therefore, Cloud-based IoT model cannot meet the strict computing time requirement in latency critical applications demanding real-time operation.Recently, Edge or Fog computing has emerged as a solution to address the drawbacks of Cloud-based IoT eHealth models. This architecture enables us to keep up with the real-time responsiveness demanded by eHealth applications by reducing the latency that arises when senor readouts travels between endpoint IoT devices and Cloud.

Although AI is the key to unlock IoT eHealth potentials, efficient techniques to distribute the intelligence across the entire IoT network from Device, and Edge to Cloud is highly required. Indeed, each of these elements (Device, Edge, and Cloud) plays an important role in the technology ecosystem, despite the proliferation of Edge and Cloud Computing today. Although this sort of vertical integration and "Collaborative" machine learning and intelligence across Device-Edge-Cloud brings tremendous benefits, it results in several design and development issues. In this paper, we propose a novel multi-layer architecture for IoT eHealth applications based on the concept of Collaborative machine learning. We investigate and examine traditional centralized Cloud-based IoT eHealth architectures and discuss how a hierarchical Device-Edge-Cloud architecture can be accurately leveraged to achieve low energy consumption, and low latency. Finally, a real-time arrhythmia monitoring and classification system is implemented as a case study to better demonstrate the details of the proposed architecture. The 


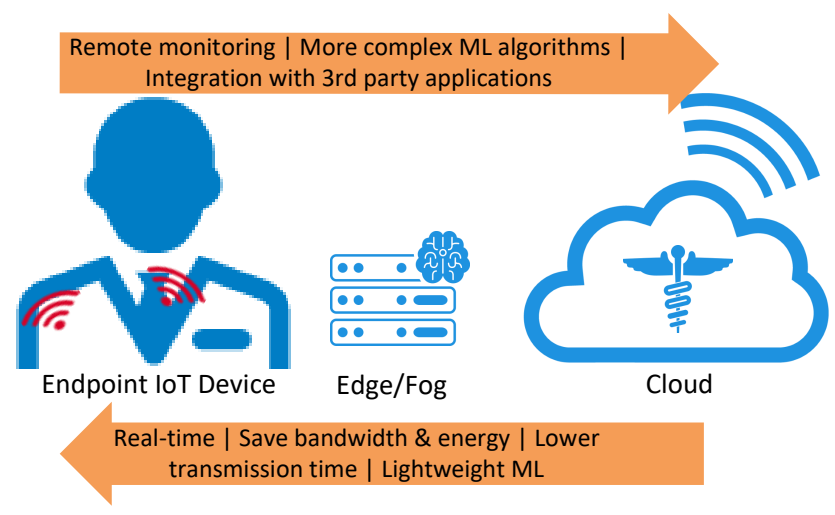

Figure 1: Overall architecture of eHealth IoT: distributed intelligence.

feasibility of taking advantage of machine learning algorithms to study the heart electrical activities has been reported in previous contributions. To name a few of them, Linear Discriminant (LD) [3], AdaBoost [12], Genetic Algorithm-Back Propagation Neural Network (GA-BPNN) [7], Multi-Layer Perceptron (MLP) [5, 8], and Support Vector Machines (SVM) $[10,15]$ have been successfully employed to analyze ECG signals. However, to the best of our knowledge, none of them has been tailored to address the requirements of IoT applications.

The rest of the paper is organized as follow: In Section II, we outline the proposed architecture. Then, we present the details of our architecture using an ECG-based arrhythmia monitoring in Section III. Evaluations and experimental results are discussed in Section IV, while Section V concludes the paper.

\section{OUTLINE OF THE PROPOSED ARCHITECTURE}

Fig. 1, outlines the general architectural elements of IoT which is partitioned into three main tiers namely, IoT eHealth Device Layer, IoT eHealth Fog Layer and IoT eHealth Cloud Layer.

IoT eHealth Device Layer. there is a vast variety of physical or virtual health sensors such as ECG/EKG monitor, heart rate monitor, glucose monitor, blood pressure monitor, body temperature monitor, hemoglobin monitor, and activity monitor $[2,13,14]$ to monitor health conditions. The rich set of IoT health devices enables individuals to monitor their health conditions in real-time, and synchronize their data securely with the Cloud.

IoT eHealth Cloud Layer: currently the status quo approach employed in most intelligent applications provided by cutting-edge solution providers is to store all sensor data in the Cloud and perform machine learning processing. The two worlds of IoT (incl. eHealth IoT) and Cloud experienced a swift and independent progress. However, the complementary features of IoT and Big Data generated many new opportunities and advantages. The main drivers for integration of IoT and Cloud are listed below:

- Communication: Cloud platform can be leveraged with the help of IoT to deliver scalable domain-independent services by providing appropriate service oriented domain mediators.
- Resource pooling: Physical resources of IoT can be integrated in Cloud resource pool enabling us to allocate and share them on-demand like regular Infrastructure as a Service (IaaS).

- Storage: IoT drives a real tsunami of big characterized by volume, variety, and velocity. In this context, IoT benefits from large-scale and long-lived storage of Cloud.

- Computation: Data processing is typically a very resource hungry task. Therefore, IoT can benefit from virtually unlimited processing resource of Cloud to aggregate data and execute batch and/or real-time analytics on the collected data.

IoT eHealth Fog Layer: by emergence of more powerful and energy efficient wearable devices and the introduction of the Edge Intelligence (EI) concept, the conventional Health IoT Cloud paradigm is transforming. In this context, we present a novel Edge/Fog driven computing architecture for Health IoT. The major benefits of Edge Intelligence include:

- Real-time: decisions can be taken more quickly and efficiently by positioning machine learning algorithms in the endpoint IoT devices or Edge nodes.

- Roundtrip delay: placing local decision makers in the Device or at the Edge results in smaller roundtrip delay on decisionmaking.

- Communication cost: rather than raw data, by a pre-processing phase at Edge, only important feedbacks, alarms, or decisions are transferred to the Cloud servers. This approach minimizes communication and message overhead, resulting in lower communication costs.

- Local policy: local regulations and access control policies can be applied better during data processing.

Although, migrating more intelligence to Edge significantly improve many tasks, several important questions arise here. In particular, how to make a tradeoff between these extremes (Edge and Cloud) and deploy advanced machine learning algoritums across the aforementioned layers. To address this, we introduce the Collaborative Intelligence concept which enables us to have the best of different worlds. Collaborative Intelligence is a novel concept which considers all major aspects of Device-Edge-Cloud Intelligence enabling us to have the best of different worlds. The key idea of Collaborative Intelligence is to distribute the intelligence across Device-Edge-Cloud to achieve an optimal solution while satisfying the given constrations.

\section{CASE STUDY: ARRHYTHMIA DETECTION}

To justify the proposed multi-layer collaborative intelligence, we present a novel online arrhythmia detection as a case study. Due to capability of Artificial Neural Networks (ANN) to achieve higher performance, they have become progressively popular as the core machine learning technique in several applications such as classification. Therefore, in this case study, we rely on ANN as the core of our intelligence. In particular, we use a shallow feed-forward neural network as machine learning on-chip and a Convolutional Neural Network $(\mathrm{CNN})$ as the core intelligence at the Edge. This flexible distributed solution enables us to compromise between Accuracy, Communication latency (transmition time), Processing time, and Energy consumption. For instance, a lightweight machine learning 
on-chip algorithm has a lower accuracy compared to complex machine learning algorithms at the Edge and in the Cloud. However, it minimizes the communication latency and energy consumption for data transmission across Device-Edge-Cloud. On the other hand, heavy computation tasks such as training the machine learning models are fully performed in the Cloud servers which have unlimited processing, memory, and storage resources. Machine learning models at the Edge/Fog nodes can be periodically updated by the Cloud to enable personalization in the decision making considering the unique features of each patient.

\subsection{Machine Learning on Chip}

For machine learning on-chip we utilize a shallow ANN with a minimum number of features to keep the final algorithm less computationalintensive, so it can be ported to a low-end IoT device to perform the detection on-demand. To detect the abnormal activity of the heart, we combined the abnormal classes of ECG signals (supraventricular ectopic beat (S), ventricular ectopic beat (V), fusion (F), and unknown beat [1]) into one group, so we trained our model by the input of two groups of data: normal beats and abnormal beats.

3.1.1 Signal Pre-processing. Signal pre-processing is an important task prior to feature extraction to remove unwanted noises from ECG recordings. In this study, the following steps have been applied to ECG signals:

- DC noise removal: first, the dc noise of the ECG signals are removed by subtracting the mean of the ECG signals from each sample point. The output of this step is an ECG signal which its amplitude is pulled back to the zero level.

- High frequency noise removal: high frequency noise can be appeared in ECG signals due to several phenomena such as patients muscle contractions during ECG monitoring. To remove high frequency noises, we use a low-pass filter (LPF) to attenuate signals with frequencies higher than the cutoff frequency.

- Low frequency noise removal: several factors such as respiration of the patient can contribute to low frequency noises. To tackle low frequency noises, we exploit a derivative based (high pass) filter to attenuate unwanted frequencies from the ECG signals.

- Power line interference removal: as studied in [4], power line interference that has $60 \mathrm{~Hz}$ pickup and harmonics can appear in the ECG signals. Therefore, to handle this unwanted noises, we use a band-stop filter to remove those harmonics from the ECG signals.

3.1.2 Feature Engineering. Because the objective of this study is to implement the ECG signal processing on an IoT wearable device, the feature extraction was performed in a way to obtain the lowest possible number of working features to train an ML model effectively.

A typical normal ECG signal is depicted in Fig. 2. First, the R-R intervals [3] are computed according to the time difference of the following beats similar to the work of Mondéjar-Guerra et al. [10]. This includes four features [10]:

- Pre-RR: backward time difference of two consequent heartbeats.

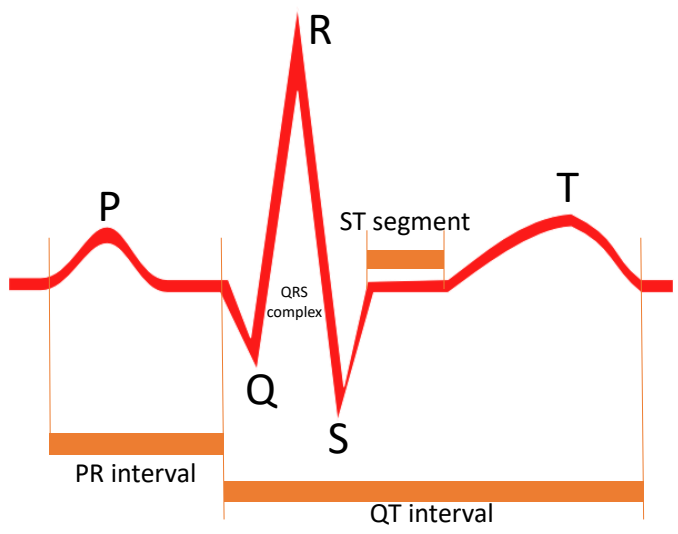

Figure 2: A typical normal ECG wave.

- Post-RR: forward time difference of two consequent heartbeats.

- Local-RR: the average of 10 consequent Pre-RR values.

- Global-RR: similar to Local-RR but for last 20 minutes instead of last 10 values.

In addition to these 4 features, we could employ their normalized values as separate features to generate 8 features as a total.

Second, Mondéjar-Guerra et al. [10] proposed a novel morphological descriptor to extract 4 features of ECG signals, which are calculated based on the distance between the R-peak and four points of the beat [10]:

- morph1: $\max ($ beat $[0,40])$.

- morph2: $\min ($ beat$[75,85])$.

- morph3: $\min ($ beat $[95,105])$.

- morph4: $\max ($ beat$[150,180])$.

Another candidate is higher-order statistics (HOS) algorithm, which groups the beats into 5 subgroups and applies HOS techniques on each one to compute skewness and kurtosis [10]. Thus, this algorithm generates 10 features. Higher order statistics has been incorporated successfully in the previous studies to extract features of the QRS complex of ECG signals [11].

Table 1 shows the tested combination of these features and the number of features in each test. This approach helped us to generate a fewer number of feature, which consequently, requires less computational resources on the low-end devices in comparison to the feature extractions found in the literature.

3.1.3 Artificial Neural Network. as mentioned, based on several experiments, a shallow artificial neural network has been selected for machine learning on chip. Fig. 3 illustrated the architecture of the ANN as an ECG classifier. The input layer processes the features $X$ and relay them to the hidden layers. At the latest layer, there is one neuron for each class $c_{i}$ and the corresponding output $Z_{c_{i}}$ is expressed as:

$$
\begin{array}{r}
Z_{c_{i}}(h, \theta)=\sum_{j=1}^{N_{h^{l}}+1} h_{j}^{(l)} \theta_{j, i}, \\
1 \leq i \leq N_{c}, \\
1 \leq l \leq N_{L},
\end{array}
$$


Table 1: Combination of feature extraction algorithms.

\begin{tabular}{cccc} 
Model No. & Combination & Number of Features & Total Features \\
\hline 1 & HOS + R-R + Norm R-R & $10+4+4$ & 18 \\
2 & R-R + Norm R-R + Custom morph & $4+4+4$ & 12 \\
3 & R-R + Custom morph & $4+4$ & 8
\end{tabular}

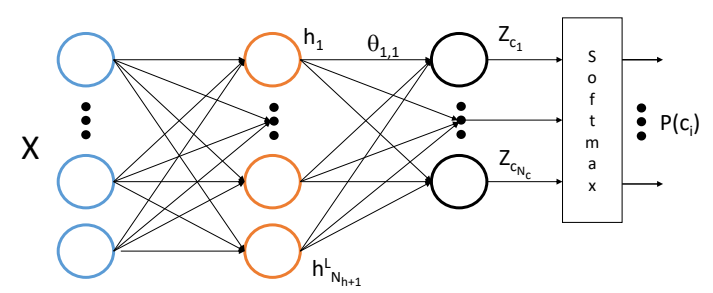

Figure 3: Machine learning on-chip.

where $h_{j}^{(l)}$ is the output of the activation function of neuron $j$ in the hidden layer $l$ and $\theta_{j, i}$ is the weight from neuron $j$ to the output class $c_{i}, N_{L}$ is the number of hidden layers, and $N_{c}$ is the number of ECG classes. Note that the outputs of ANN are connected to a softmax activation function to compute the probability of each class $P\left(c_{i}\right)$ :

$$
P\left(c_{i} \mid h, \theta\right)=\frac{e^{Z_{c_{i}}(h, \theta)}}{\sum_{j=1}^{N_{c}} e^{Z_{c_{j}}(h, \theta)}}
$$

It is worth mentioning that finding an appropriate number of hidden layers and number of neurons in each hidden layer is very important in order to achieve a good accuracy, while keeping the Power-Performance-Area (PPA) overhead low.

3.1.4 When to Switch from Device to Edge. as mentioned before, the output of the ANN as our on-chip ECG classifier is connected to a softmax function. The probability vector obtained at the end of the ANN network can be interpreted as the model confidence scores. If $P\left(c_{i}\right)$ estimates the certainty of the ANN model for class $c_{i}$, we can decide to accept the predicted result or reject the machine learning on-chip opinion and ask the Edge node to provide its opinion as follow:

$$
\begin{cases}\text { Accept on-chip opinion } & \text { if } \max _{i \in n}\left(P\left(c_{i} \mid h, \theta\right) \geq \tau\right. \\ \text { Reject and ask Edge } & \text { if } \max _{i \in n}\left(P\left(c_{i} \mid h, \theta\right)<\tau\right.\end{cases}
$$

Notice that the decision threshold $\tau$ is sensitive to Covariate Shift. As dataset change, the distribution of ECG features used as predictors (covariates) shifts from the training phase to the production phase. This implies that when the mean of the data is largely differ from the training set, ANN cannot provides high confidence scores. To tackle this critical issues, it is very important to accurately perform the pre-processing phase to calibrate the input features.

\subsection{Edge Intelligence}

Leveraging Edge devices enables delay-sensitive mission-critical applications to make online real-time decisions. Patient anomaly
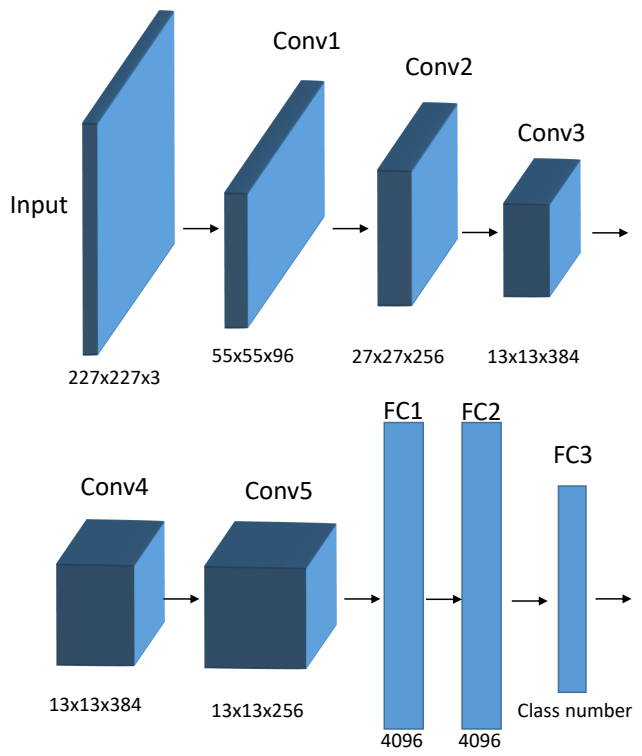

Figure 4: CNN layers for intelligence at the Edge.

recognition and heath monitoring applications can be placed in a category in which transferring a huge volume of streaming health data (such as ECG signals) on wireless networks. Development of more powerful and energy efficient System-on-a-Chip (SoC), in particular for mobile devices, allows more computing tasks and more complex machine learning algorithms to take place on the Edge nodes. Convolutional Neural Networks (CNN) are a class of deep neural networks that have been proven to be very effective in several areas such as image classification. Inspiring by this observation, we extending ECG signal processing to the Edge in addition to shallow footprint machine learning on-chip. To do so, we exploit a $\mathrm{CNN}$ to be able to improve the performance of the arrhythmia detection.

3.2.1 Pre-processing. both $2 \mathrm{D}$ CNNs and $1 \mathrm{D}$ CNNs can be used for the classification of ECG signals. In this study, we rely on 2D CNNs to be able to identify arrhythmia. Since the input to a CNN is an image, we transform each beat of the ECG signals to an image of size 227-by-227-by-3. Deep learning and in particular image processing based on CNN is typically relevant when there is a huge amount of data available. As will be discussed later, the ECG benchmark database is imbalanced, meaning that classes are not represented equally. To overcome these issues, we use resampling and data augmentation techniques. We will "augment" our ECG classes via a number of random transformation of images such as rotation, zooming and cropping. This helps to tackle overfitting issues and leads to better generalization. 
3.2.2 CNN Architecture. CNNs are inspired by the biological process of visual cortex in which each neuron is sensitive to stimuli of just a confined area of an image. Note that an individual area can partially overlap with the area of near neurons to cover and process the whole image. One of the key advantages of $\mathrm{CNN}$ is that they do not rely on traditional hand-engineered features. This eliminates the need of having any prior knowledge in feature engineering to be able to design an efficient image processing system.

- CNN layers: in this study, to identify arrhythmia, we use a CNN consisting of five 2D convolution layers, three max pooling layers, and three fully connected layers. Fig. 4 illustrates our $\mathrm{CNN}$ architecture including the filter size, stride, and padding. Note that our CNN model is based on Alexnet [6].

- Activation function: the most common activation functions are Sigmoid, Tanh, and the Rectified Linear Unit (ReLU). In general, Sigmoid has two shortcomings. First, Sigmoid potentially can saturate and thus kill gradients. Second, the output of Sigmoid is not zero-centered. Unlike Sigmoid, Tanh is zero-centered, however it tends to vanish gradients. To tackle the aforementioned issues, in this study, we use ReLU activation in neurons.

- Regulization: there are several standard ways to improve generalization and prevent overfitting in neural networks. In this study, we leverage the Dropout mechanism i.e., ignoring some random neurons in a particular forward and backward pass during the training phase.

- Covariate shift: to handle internal covariate shift in the CNN, we apply batch normalization by normalizing the output of the previous activation layer. This approach enables each $\mathrm{CNN}$ layer to learn more independently of other layers.

\subsection{Big Data Analytics in the Cloud}

Although we outsourced the decision-making task from the Cloud nodes to endpoint IoT devices and Fog nodes, it has been proved that still training a deep machine learning is a huge computational burden for such devices. Considering this fact, training the machine learning on-chip model as well as CNN-based machine learning model at the Edge are fully positioned in the eHealth Cloud nodes. The Cloud also empower us to build progressive machine learning algorithms over time customized for each patient. Machine learning models are periodically trained and updated over time with the new collected data and feedback. Next, the extracted model weights for each and every layer are sent back to the corresponding endpoint and Edge devices to provide online real-time decision making. Such periodical monitoring and re-training of the machine learning models enable personalization in the decision making tailored for each individual patient. In addition, Cloud improves the understanding of health providers of the evolutionary changes of diseases in each patient.

\section{EXPERIMENTAL RESULTS}

\subsection{Experimental Setup}

In this study, the well-known MIT-BIH database is used to train an ML model to predict the abnormal situations in heart electrical activities [9]. This database contains 48 ECG records of about 30 minutes, sampled at $360 \mathrm{~Hz}$ with 11-bit resolution from 47 different patients, and as a result, the database contains 110,000 beats approximately. Unfortunately, the database is highly imbalanced, and this fact is one of the important challenges in training an ML model with a good level of precision and recall using this database. An approximation of $90 \%$ of the beats belongs to the normal class, and only $3 \%, 6 \%$, and $1 \%$ of the beats are available in the supraventricular ectopic beat $(\mathrm{S})$, ventricular ectopic beat $(\mathrm{V}), \mathrm{fu}-$ sion (F) classes, respectively. As recommended by the AAMI, the records with paced beats $(102,104,107$, and 217) should not be considered. The remaining records were divided into two sets using the inter-patient scheme [3], for the purpose of training and validation. Each dataset contained 22 patient with similar proportions of beat types.

To evaluate the combination of features and choosing the proper classification method, we trained various classifiers using the training feature set and tested them using the evaluation set. The best results in the previous ML studies of MIT-BIH database were achieved using SVM models [10], so to compare our results, we trained an SVM model similar to the work of Mondéjar-Guerra et al. [15].

\subsection{Evaluation Metrics}

In machine learning algorithms, precision or positive predictive value is the ratio of correctly predicted classes (here normal or abnormal classes) to the total predicted positive observations of that class. In other words, precision says how many of the selected objects were correct. Recall (Sensitivity) is the ratio of correctly predicted positive observations to all the observations in that actual class, which means how many of the objects that should have been selected were actually selected. F1 score is the weighted average of precision and recall. Accuracy is a ratio of correctly predicted observations to the total observations.

There are well known categories for the output of a classification algorithm: True Positives (TP), True Negatives (TN), False Positives (FP), and False Negatives (FN). With these in mind, the definition of the above metrics would be as follow:

$$
\begin{array}{r}
\text { Accuracy }=\frac{T P+T N}{T P+F P+F N+T N}, \\
\text { Precision }=\frac{T}{T P+F P}, \\
\text { Recall }=\frac{T P}{T P+F N}, \\
F 1 \text { Score }=\frac{2 \times(\text { Recall } \times \text { Precision })}{\text { Recall }+ \text { Precision }} .
\end{array}
$$

\subsection{Evaluation}

Due to the final goal of the current project to port the code to an IoT wearable device in order to detect dangerous heart performance, we combined the 3 classes of abnormal beat types into one class, so we had two groups of beats to label the data (instead of 4): normal and abnormal beats, in which the abnormal beat class comprises the S, V, and F beats. Figure 5 represents the waveform of these 2 classes. This also helped us to overcome the problem of the imbalance of the studied database. This 2-class dataset was the input of the ANN model. 


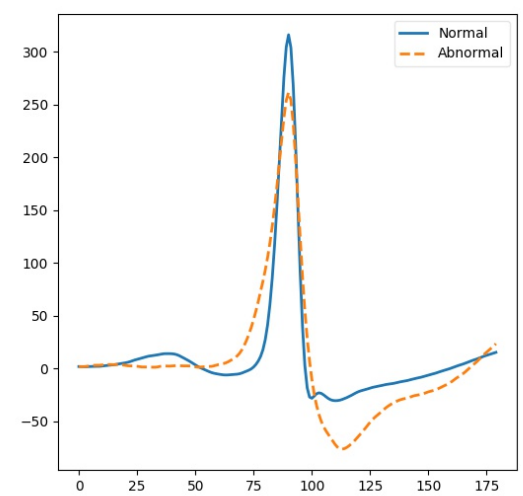

Figure 5: 2 classes of ECG signals: normal and abnormal.

Table 2: Confusion Matrix of the 4-class ANN model no. 1.

\begin{tabular}{|c|c|c|c|c|}
\cline { 2 - 5 } \multicolumn{1}{c|}{} & Predicted N & Predicted S & Predicted V & Predicted F \\
\hline Actual N & 44019 & 0 & 14 & 0 \\
\hline Actual S & 1817 & 0 & 233 & 0 \\
\hline Actual V & 529 & 0 & 2691 & 0 \\
\hline Actual F & 314 & 0 & 74 & 0 \\
\hline
\end{tabular}

Table 3: Precision of the 2-class models.

\begin{tabular}{|c|c|c|c|}
\cline { 2 - 4 } \multicolumn{1}{c|}{} & Model no. 1 & Model no. 2 & Model no. 3 \\
\hline Normal & 0.95 & 0.95 & 0.95 \\
\hline Abnormal & 0.92 & 0.86 & 0.98 \\
\hline
\end{tabular}

Table 4: Sensitivity of the 2-class models.

\begin{tabular}{|c|c|c|c|}
\cline { 2 - 4 } \multicolumn{1}{c|}{} & Model no. 1 & Model no. 2 & Model no. 3 \\
\hline Normal & 0.99 & 0.99 & 1.00 \\
\hline Abnormal & 0.60 & 0.56 & 0.61 \\
\hline
\end{tabular}

To obtain the ANN model configuration, an iterative approach was employed to test various configurations and measure the output error. The optimum configuration (both in error and computational resource requirement) contained three layers with 100 neurons in the hidden layer, Rectifier Linear Unit (reLU) activation function, a constant learning rate of 0.001 , and $\alpha=0.0001$. The trained models were used to detect the type of beats in the 4-class evaluation dataset. We used the ANN model to train the state-ofthe-art 2-class models. The trained models were used to detect the type of beats in the 4-class evaluation dataset.

The precision of the 2-class ANN models is listed in Table 3 for each of the 2 classes of beats. Similarly, the sensitivity of the trained models is represented in Table 4.

The model trained with 8 features (model no. 3) showed a high level of accuracy, precision, and sensitivity in comparison to the other models, and it has a fewer number of features, which results in a less computational intensive code to be ported to the low-end IoT device. The correlation of these 8 features is presented in Figure

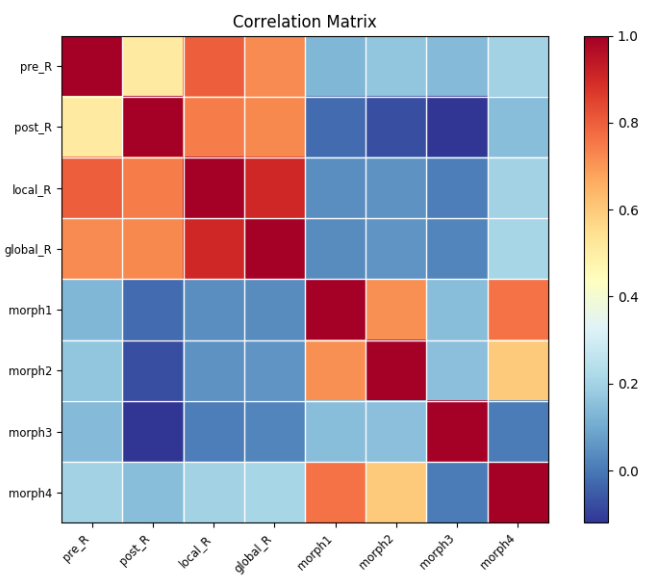

Figure 6: Correlation matrix of 8 selected features to be deployed to the IoT device.

Table 5: Confusion Matrix of the 2-class model no. 3.

\begin{tabular}{|c|c|c|}
\cline { 2 - 3 } \multicolumn{1}{c|}{} & Predicted normal & Predicted abnormal \\
\hline Actual normal & 43947 & 86 \\
\hline Actual abnormal & 2216 & 3442 \\
\hline
\end{tabular}

Table 6: Performance of the 2-class ANN model no. 3.

\begin{tabular}{|c|c|c|c|}
\cline { 2 - 4 } \multicolumn{1}{c|}{} & Precision & Sensitivity & F1-score \\
\hline Normal & 0.95 & 1.00 & 0.97 \\
\hline Abnormal & 0.98 & 0.61 & 0.75 \\
\hline Avg / Total & 0.95 & 0.95 & 0.95 \\
\hline
\end{tabular}

6. Table 5 shows the confusion matrix of the output of the evaluation of this model, and the precision, sensitivity, and F1 score of each of the two classes are represented in Table 6 . The achieved accuracy of the trained model evaluated on the test dataset is $95 \%$, which is higher than the similar studies performed using traditional SVM models $[10,15]$. In comparison to those studies, we combined the beats categories regarding the final application of the code, instead of using an ensemble of trained models, which is not suitable for low-end IoT devices due to their computational power. The overall accuracy of similar models is $94 \%$ (ensemble of SVMs to overcome the problem of the imbalance of database) with the precision of $66 \%$ and sensitivity of $70 \%$ in the work of MondéjarGuerra et al. [15], or the accuracy of $88 \%$ with the precision of $60 \%$ and sensitivity of $86 \%$ in the work of Zhang et al. [10]. We also classified the ECG signals using the introduced CNN in an edge node to be able to evaluate its performance. The results show that accuracy and sensitivity of the CNN model is $98 \%$ and $96 \%$, respectively which are very promising.

\section{CONCLUSIONS}

The massive wave of Intelligent IoT-based innovation can extend the boundaries of healthcare outside of hospital settings by transforming the hospital-centric to patient-centric ecosystem. In this 
paper, we proposed a holistic hierarchical eHealth architecture including wearable Device layer, Edge/Fog layer, and Cloud layer to continuously monitor health related information of subjects and provide real-time insights to health providers anywhere at any time. The key advantage of such a collaborative solution and intelligence is to compromise between Accuracy, Communication latency (transmition time), Processing time, and Energy consumption. We also presented a case study to provide an illustrative example on how to deploy advanced machine learning techniques such as Convolutional Neural Network to this novel architecture.

\section{REFERENCES}

[1] 1998. Testing and Reporting Performance Results of Cardiac Rhythm and ST Segment Measurement Algorithms, Association for the Advancement of Medical Instrumentation. Technical Report.

[2] Nicholas Constant, Orrett Douglas-Prawl, Samuel Johnson, and Kunal Mankodiya. 2015. Pulse-Glasses: An unobtrusive, wearable HR monitor with Internet-of-Things functionality. In Wearable and Implantable Body Sensor Networks (BSN), 2015 IEEE 12th International Conference on. IEEE, 1-5.

[3] P. de Chazal, M. ODwyer, and R. B. Reilly. 2004. Automatic classification of heartbeats using ECG morphology and heartbeat interval features. IEEE Trans. Biomed. Eng 51, 7 (2004), 1196囚1a71206.

[4] Ali Isin and Selen Ozdalili. 2017. Cardiac arrhythmia detection using deep learning. Procedia Computer Science 120 (2017), 268-275.

[5] H. Khorrami and M. Moavenian. 2010. A comparative study of DWT, CWT and DCT transformations in ecg arrhythmias classification. Expert Syst. Appl. 37, 8 (2010), 5751-5757.
[6] Alex Krizhevsky, Ilya Sutskever, and Geoffrey E Hinton. 2012. Imagenet classification with deep convolutional neural networks. In Advances in neural information processing systems. 1097-1105.

[7] H. Li, D. Yuan, X. Ma, D. Cui, and L. Cao. 2017. Genetic algorithm for the optimization of features and neural networks in ECG signals classification. Technical Report.

[8] R. J. Martis, U. R. Acharya, K. Mandana, A. Ray, and C. Chakraborty. 2012. Application of principal component analysis to ECG signals for automated diagnosis of cardiac health. Expert Syst. Appl. 39, 14 (2012), 11792-11800.

[9] MIT-BIH, available at https://www.physionet.org/physiobank/database/mitdb/. [n. d.].

[10] V. Mondéjar-Guerra, J. Novo, J. Rouco, M. G. Penedo, and M. Ortega. 2019. Heartbeat classification fusing temporal and morphological information of ECGs via ensemble of classifiers. Biomed Signal Process Control 47 (2019), 41-48.

[11] S. Osowski and T. H. Linh. 2001. ECG beat recognition using fuzzy hybrid neural network. IEEE Trans. Biomed. Eng 48, 11 (2001), 1265囚1a71271.

[12] K. N. Rajesh and R. Dhuli. 2018. Classification of imbalanced ECG beats using re-sampling techniques and AdaBoost ensemble classifier. Biomed Signal Process Control 41 (2018).

[13] Mohd Fadlee A Rasid, WMW Musa, NAA Kadir, Anas M Noor, Farid Touati, W Mehmood, L Khriji, A Al-Busaidi, and Adel Ben Mnaouer. 2014. Embedded gateway services for Internet of Things applications in ubiquitous healthcare. In Information and Communication Technology (ICoICT), 2014 2nd International Conference on. IEEE, 145-148.

[14] Geng Yang, Li Xie, Matti Mantysalo, Xiaolin Zhou, Zhibo Pang, Li Da Xu, Sharon Kao-Walter, Qiang Chen, and Li-Rong Zheng. 2014. A health-IoT platform based on the integration of intelligent packaging, unobtrusive bio-sensor, and intelligent medicine box. Industrial Informatics, IEEE Transactions on 10, 4 (2014), 2180-2191.

[15] Z. Zhang, J. Dong, X. Luo, K. S. Choi, and X. Wu. 2014. Heartbeat classification using disease-specific feature selection. Comput. Biol. Med. 46 (2014), 789-791. 